\title{
Clinical application of whole-exome sequencing: A retrospective, single-center study
}

\author{
QIANG ZHANG, ZAILONG QIN，SHANG YI，HAO WEI，XUN ZHAO ZHOU and JIASUN SU \\ Laboratory of Genetic and Metabolism, Department of Paediatric Endocrine and Metabolism, \\ Maternal and Child Health Hospital of Guangxi, Nanning, Guangxi 530000, P.R. China
}

Received April 29, 2020; Accepted August 26, 2020

DOI: $10.3892 /$ etm.2021.10185

\begin{abstract}
The aim of the present study was to assess the practical diagnostic value of whole-exome sequencing (WES) in patients with different phenotypes and to explore possible strategies to increase the capability of WES in identifying disease-causing genes. A total of 1,360 patients (aged from 1 day to 42 years old) with manifestations of genetic diseases were genotyped using WES and statistical analysis was performed on the results obtained. Within this cohort, the overall positive rate of identification of a disease-causing gene alteration was $44.41 \%$. The positive identification rate where trio-samples were used (from the proband and both parents) was higher than that where a single proband sample was used (50.00 vs. $43.71 \%$ ), and 604 positive cases with 150 genetic syndromes, 510 genes and 718 mutations were detected. Missense mutations were the most common variations $(n=335$, $45.27 \%$ ) and visual or auditory abnormalities (58.51\%) had the highest rate of association with a genetic abnormality. The positive detection rate of WES was elevated with the increase in the number of clinical symptoms from 1 to 8 . The present study indicated that WES may be used as a valuable tool in the clinic and the positive rate depends more on the professional experience of clinicians rather than on the analytical capabilities of the data analyst. At the same time, particular attention must be paid to certain possible factors (such as the age of the patients as well as possible exon deletions), which may affect the diagnostic rate while applying this process.
\end{abstract}

\section{Introduction}

Based on World Health Organization estimates, China's birth defect rate is $5.6 \%$ at present, which is significantly higher than

Correspondence to: Professor Qiang Zhang, Laboratory of Genetic and Metabolism, Department of Paediatric Endocrine and Metabolism, Maternal and Child Health Hospital of Guangxi, 59 Xiangzhu Road, Nanning, Guangxi 530000, P.R. China E-mail: 450293052@qq.com

Key words: whole-exome sequencing, pathogenicity assessment, phenotypic abnormality, human phenotype ontology the birth defect rate of $4.72 \%$ in developed countries (1). In addition, birth defects have risen from fourth place in 2000 to 2nd place in 2011 in the league table of child mortality causes in China, accounting for $19.10 \%$ of child deaths (2). In the 21st century, the development of genetic testing technology and the ability to reveal the causes of genetic diseases have promoted the revolutionary transformation of medical practice from the symptoms-based diagnostics of traditional medicine to the cause-based diagnostics of modern medicine (3).

With the large population in China and the increasing number of patients carrying genetic diseases, inadequate numbers of qualified genetic professionals and testing capabilities represent significant challenges (1). The diagnosis and treatment of inherited metabolic disorders has become an important task for clinicians $(4,5)$. In 2009, $\mathrm{Ng}$ et al (6) first applied next-generation sequencing to capture the sequences of human genome exons in order to analyze the pathogenic variations of single-gene disorders. Since then, the technique of whole-exome sequencing (WES) has been widely used, due to its ability to provide potential molecular genetic proof for genetic diseases in suspected cases after clinical assessment (7).

Guangxi, a province located in southwestern China, has a population including several ethnic minorities that is genetically heterogeneous (8). Based on our experience, the application of WES in the local population with a genetic disease presentation has proven to be beneficial. Guangxi Maternal and Child Health Hospital (Nanning, China) is one of the leading hospitals responsible for the diagnosis and treatment of genetic diseases in this region (9). In the present study, the data of 1,360 cases of WES in a single center were reviewed and summarized to assess the practical diagnostic value of WES and to explore how to improve the ability of this technique to find disease-causing genes.

\section{Patients and methods}

Basic information. A total of 1,360 cases subjected to WES at the Guangxi Maternal and Child Health Hospital (Nanning, China) between January 2017 and July 2019 were reviewed. Their age ranged from 1 day to 42 years $(4.72 \pm 7.67)$ and the ratio of males to females was 1.74:1. Among them, 456 were inpatients and 904 were outpatients. The clinical characteristics of all patients were evaluated. The patients' 
ethnicities included Han, Zhuang, Yao, Uygur, Miao as well as other ethnic minorities which is representative of the ethnic composition of this region (10). Therefore, the present cohort may be considered as a representative sample. The present study was a retrospective study, and the data were collected as part of the routine clinical procedure and no informed consent is required. However, each patient or guardians signed the informed consent before performing the WES. The publication of the article is approved by the Ethics Committee of Guangxi Zhuang Autonomous Region Maternal and Child Health Hospital and the Children's Hospital (Reference File No.:2017, [2-11]).

\section{WES and biometric filtering}

DNA extraction. Blood samples (2 $\mathrm{ml}$ of peripheral blood) were collected from the patients and their parents for WES. Genomic DNA was isolated using the Lab-Aid DNA kit (Zeesan Biotech Co., Ltd) and stored at $-80^{\circ} \mathrm{C}$.

WES and copy number variation $(C N V)$ analysis. Human exome sequencing libraries were constructed using the Agilent SureSelect Human All Exon V5 kit (Agilent Technologies, Inc.), and amplicons were generated and sequenced with the Illumina HiSeq 2500 system (Illumina, Inc.). All procedures were performed according to the manufacturer's instructions. After sequencing, reads were aligned to an indexed human reference genome (GRCh37/hg19) with Burrows-Wheeler transformation 0.7.15-r1140 (11). Duplicate reads were removed using Picard v.1.85 (http://picard.sourceforge.net) prior to further processing. Base recalibration and variant calling were performed using the Genome Analysis Toolkit v.2.3-4Lite (12). Finally, identified variants were saved in a variant call format. In addition, in the present study, there was an attempt to reveal CNVs with a read depth-based CNV detection method (13-16). The detection methods can be separated into four steps: Raw coverage normalization, correction for sample-specific coverage biases, CNV calling, and partially-mapped read analysis and all copy number variants were finally confirmed by Illumina Human Cyto SNP12 kit. Translational Genomics Expert (LifeMap Sciences, Inc.) was used for variant prioritization. Integrative Genomics Viewer (IGV; v.2.4.15; http://software.broadinstitute.org/software/igv/) was used to visualize WES data and assess the coverage of the exons.

Quality control. The capture area of WES was 60M. The average sequencing depth of each sample was $>120 \mathrm{X}$ and produced $12 \mathrm{G}$ clean data. At the same time, the actual qualified data output of the library was $>95 \%$ with an average Q $30 \geq 80 \%$ for each sample and the accurate coverage of the exon region $(>20 X)$ should be $\geq 97 \%$; base type distribution was uniform with no GC separation. Sanger sequencing was used to verify the mutations and their origins.

In order to reduce the interference of noise signals in the results, a series of measures was implemented. First, in the experimental process, each sample was detected under the same experimental conditions to reduce any intra-batch differences. Furthermore, normalization of WES data was performed to avoid any differences caused by the capture or sequencing processes. In addition, mass correction on the exome region of the sample was performed including a GC correction. Finally, biological information processing on the same exome region of different samples on the same batch was routinely performed.

Sequencing data analysis. The Genome Analysis Toolkit (GATK) was used for variant calling (GATK HaplotypeCaller). The TGex software version 3.4.1 (LifeMap Sciences) was used to annotate the selected single nucleotide variants (SNVs) and indels. 'Rare deleterious' mutations were defined as those that met the following criteria: a) They led to a stop-gain, stop-loss, nonsynchronous, frameshift or splice-site mutation and (b) the reference genome GRCh37/hg19 from the Genome Reference Consortium was used (https://www.ncbi.nlm.nih.gov/grc).

Interpretation of variations. Pathogenicity assessment was based on the American College of Medical Genetics and Genomics (ACMG)/Association for Molecular Pathology (AMP) 2015 guidelines and the variants were classified as either 'benign', 'likely benign', 'uncertain significance', 'likely pathogenic' or 'pathogenic' based on InterVar (http://wintervar. wglab.org/) (17).

Statistical analysis. Data analysis was conducted by $\chi^{2}$ test using the IBM SPSS version 19.0 statistical analysis software. $\mathrm{P}<0.05$ was considered significant. $\chi^{2}$ test was used to compare the frequencies of positive (disease-related genes found)/negative (No association was found between disease and gene) detection among medical staff.

\section{Results}

Baseline characteristics. In the present study information and WES data was collected on 1,360 patients from the Pediatric Intensive Care Unit and the Pediatrics, Eugenics, Child Rehabilitation, Neonatal, Otolaryngology and General Pediatric Inpatient Departments as well as the Surgery Clinic of Guangxi Maternal and Child Health Hospital (Nanning, China) between January 2017 and July 2019. Of the 1,360 patients, 456 were hospitalized and 904 were out-patients. The positive rates (detection rate of disease-associated genes) of hospitalized and out-patients were $43.20 \%$ (198/456) and $45.02 \%$ (407/904), respectively. Among all of the cases, 1,217 were single-sample patients (where only the proband was tested) and 144 were trio-samples (the proband and their parents). Of the 1,217 single-sample patients, 532 were positive (43.71\%), while 72 positive samples were detected within 144 family samples (50.00\%). Among the 604 positive patients, including 532 single and 72 family samples, 89 cases had CNVs with an associated positive rate of $14.74 \%, 83.28 \%$ were the non-CNVs and $1.98 \%$ (114 cases) carried compound heterozygous mutations. The deletions of one or more exons with merge point mutations accounted for 10 cases and CNV merged with SNV accounted for 6 cases. Among them, 478 mutations had been reported previously and 240 mutations were novel (unpublished). A total of 198 mutations were de novo variations. A flow chart of the analysis of all of the cases studied is presented in Fig. 1.

Genetic variants and pathogenicity assessment. A total of 150 genetic syndromes, involving 510 genes (with respect to abnormal CNVs and point mutations) and 718 variations 
Table I. Positive rate under different clinical symptoms.

\begin{tabular}{|c|c|c|c|}
\hline Phenotypic abnormality & $\mathrm{HPO}$ & Number of subjects tested & Positive cases, $\mathrm{n}(\%)$ \\
\hline Growth abnormality & HP:0000002 & 239 & $118(49.37)$ \\
\hline \multicolumn{4}{|l|}{ Abnormality of the nervous system } \\
\hline Seizures & HP:0001250 & 229 & $102(44.54)$ \\
\hline Intellectual disability & HP:0001249 & 104 & $38(36.54)$ \\
\hline Delayed speech and language & HP:0000750 & & \\
\hline Development or autistic behavior & HP:0000729 & 116 & $38(32.76)$ \\
\hline Global developmental delay & HP:0001263 & 93 & $51(54.84)$ \\
\hline Cerebral palsy & HP:0100021 & 143 & $59(41.26)$ \\
\hline Motor deterioration & HP:0002333 & 206 & $99(48.06)$ \\
\hline Abnormality of the respiratory system & HP:0002086 & 165 & $67(40.61)$ \\
\hline \multicolumn{4}{|l|}{ Abnormality of the genitourinary system } \\
\hline Abnormality of the genital system & HP:0000078 & 182 & $66(36.26)$ \\
\hline Abnormality of the urinary system & HP:0000079 & 195 & $66(33.85)$ \\
\hline Abnormality of the skeletal system & HP:0000924 & 215 & $116(53.95)$ \\
\hline Abnormality of head or neck & HP:0000152 & 290 & $161(55.52)$ \\
\hline Abnormality of the skin & HP:0000951 & 108 & $58(53.70)$ \\
\hline Abnormality of the endocrine system & HP:0000818 & 225 & $112(49.78)$ \\
\hline \multirow[t]{2}{*}{ Hearing or vision abnormality } & HP:0000364 & 94 & $55(58.51)$ \\
\hline & HP:0000504 & & \\
\hline Abnormality of blood and blood-forming tissues & HP:0001871 & 75 & $27(36.00)$ \\
\hline Abnormality of the digestive system & HP:0025031 & 52 & $14(26.92)$ \\
\hline Abnormality of the cardiovascular system & HP:0001626 & 125 & $54(43.20)$ \\
\hline
\end{tabular}

HPO, human phenotype ontology which provides a standardized vocabulary of phenotypic abnormalities encountered in human disease. Positive cases, detection rate of disease-associated genes.

were detected in the 604 positive cases, of which 89 were CNVs, including variations associated with deletions and duplications. Based on the ACMG/AMP 2015 guidelines, 718 mutations were classified as being pathogenic (65.13\%), likely pathogenic $(25.05 \%)$ and as having uncertain significance $(9.82 \%)$. Even if certain genes may be consistent with clinical manifestations, they may not have been extensively studied and may only be identified as variants of uncertain significance. In such instances, the progress of research requires them to be tracked on a regular basis to determine whether the rating may be updated in order to provide patients with treatment and genetic counseling. At present, the 'consensus recommendations for the clinical application of genetic testing for children's genetic diseases' recommends that only pathogenic and likely pathogenic mutations may be used for prenatal or preimplantation diagnosis (18).

Classification of clinical symptoms and statistics. Among the patients, certain probands had a single symptom while others had multiple systemic phenotypes. The patients were divided into 20 categories according to their major clinical symptoms. The positive detection rate for each clinical manifestation is presented in Table I. The major clinical manifestations included short stature, motor deterioration, language development retardation, mental retardation, skeletal deformity, seizures and gonadal dysgenesis or abnormal genital organs. In order to investigate whether the positive rate of WES increased with the number of clinical symptoms, the number of clinical symptoms exhibited by each of the subjects was determined, and according to those numbers, the patients were assigned to categories 1-9 and the positive rates in those groups were then compared in a graph. Through analysis and comparison, it was determined that the positive rate of WES increased with the number of clinical symptoms in the range of 1 to 8 . The positive rate was $\sim 40 \%$ when the number of symptoms was $1-3$, but when the number of symptoms was $4-8$, the positive rate was $\sim 50 \%$ (Fig. 2). It is recommended, wherever possible, that the interpretation of WES data should be accompanied by clinical information (19). In the positive cases, the incidence of autosomal variation was 4.59 times [491 (autosomal variation cases): 107 (sex chromosomes variation cases)] that of sex chromosome abnormality. The patients with sex chromosome abnormalities were mainly male children ( $<14$ years old) and the male-to-female ratio was 15.75:1. Clinical manifestations of sex chromosome abnormalities included cryptorchidism, micro-penis and hypospadias. There were 629 non-CNVs, namely nonsense, missense, frameshift, splicing, in-frame deletions, in-frame duplications and exon-deletions. The proportion of each variant is presented in Fig. 3. In order to determine whether the capabilities of clinicians and data analysts affect the positivity rate of WES, a pediatric outpatient department, which has the largest number of test samples, was also selected to observe whether there were 


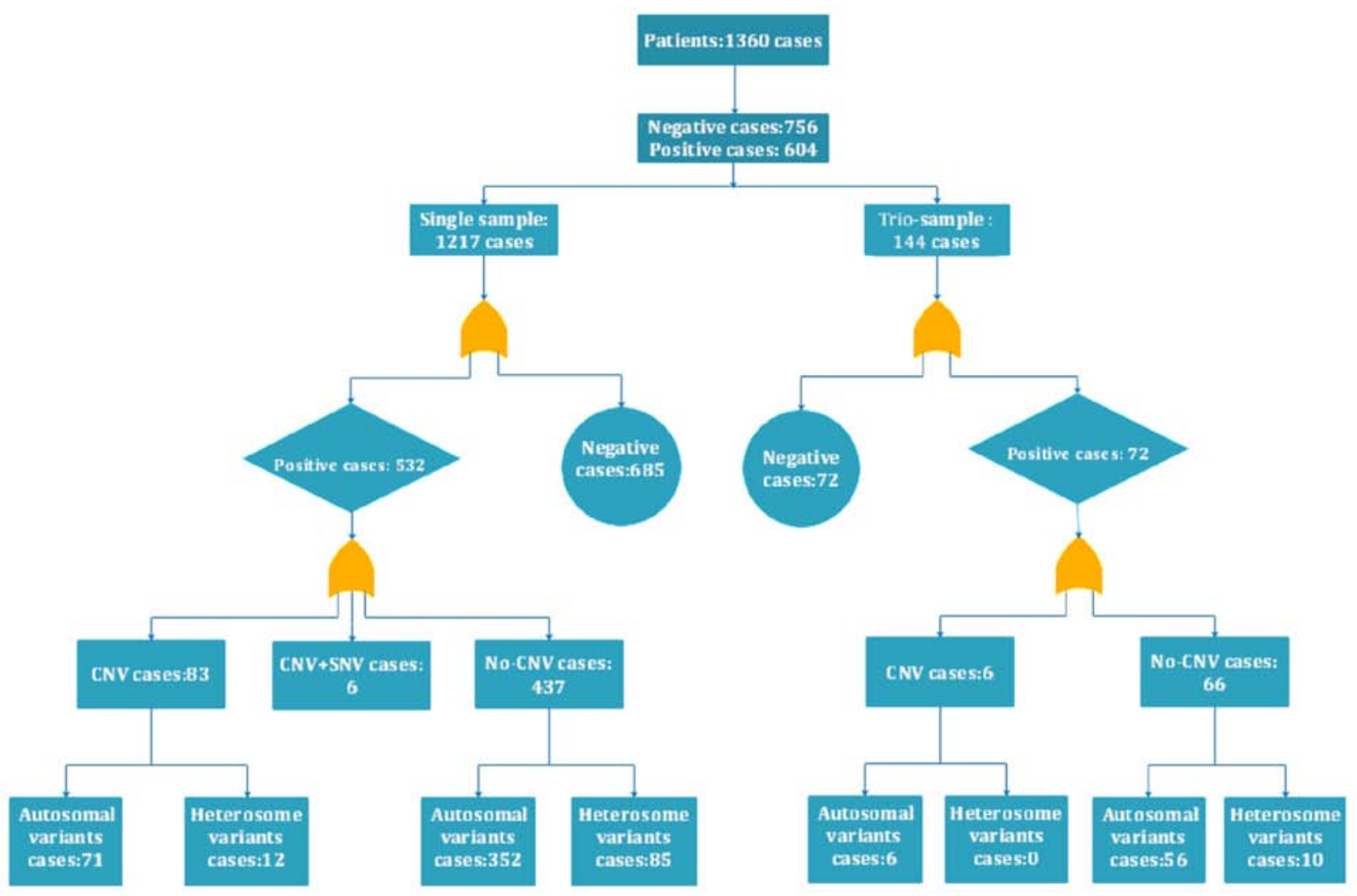

Figure 1. Test results of the 1,360 subjects. CNV, copy number variant; SNV, single nucleotide variant.

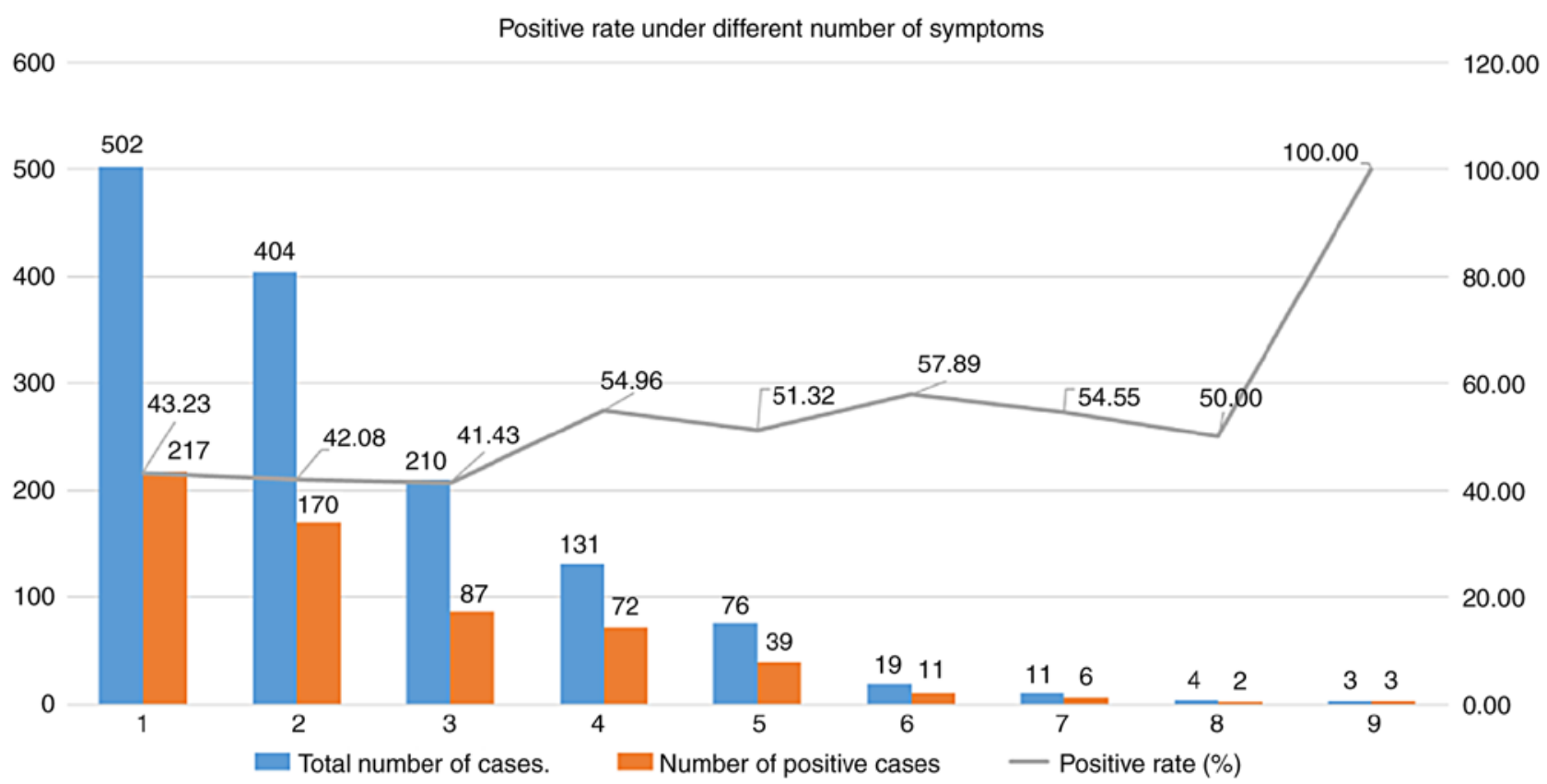

Figure 2. Positive rates for different numbers of symptoms.

any differences in the positive status of the samples submitted by individual doctors. The positive detection rates among the samples analyzed by the data analysts were also determined. As presented in Table II, different clinicians had different professional levels of expertise and experience to evaluate patients and this directly affected the WES-positive rate, but there was no significant difference between different data analysts.

\section{Discussion}

In general, Mendelian (single-gene) diseases are considered to be rare, occurring at a rate of 40-82 cases per 1,000 live births, with an estimated 7.9 million infants being born annually with serious birth defects of genetic or partially genetic origin $(20,21)$. The US Food and Drug Administration has 
Table II. Clinicians and data analysts' ability to influence the impact of whole-exome sequencing results.

\begin{tabular}{|c|c|c|c|c|c|}
\hline Staff group/ID & Positive cases & Negative cases & Positive rate $(\%)$ & Chi-square & P-value \\
\hline Clinicians & & & & 14.43 & 0.025 \\
\hline $\mathrm{A}$ & 140 & 194 & 0.42 & & \\
\hline $\mathrm{B}$ & 32 & 17 & 0.65 & & \\
\hline $\mathrm{C}$ & 21 & 34 & 0.38 & & \\
\hline $\mathrm{D}$ & 12 & 19 & 0.39 & & \\
\hline $\mathrm{E}$ & 4 & 8 & 0.33 & & \\
\hline $\mathrm{F}$ & 8 & 12 & 0.40 & & \\
\hline $\mathrm{G}$ & 1 & 7 & 0.13 & & \\
\hline Data analysts & & & & 3.46 & 0.75 \\
\hline A1 & 127 & 150 & 0.46 & & \\
\hline B1 & 74 & 82 & 0.47 & & \\
\hline $\mathrm{C} 1$ & 23 & 33 & 0.41 & & \\
\hline D1 & 18 & 23 & 0.44 & & \\
\hline E1 & 89 & 94 & 0.49 & & \\
\hline $\mathrm{F} 1$ & 14 & 24 & 0.37 & & \\
\hline G1 & 70 & 69 & 0.50 & & \\
\hline
\end{tabular}

A-G represents different clinicians, and A1-G1 different analysts. Positive cases (as diagnosed by clinicians) are defined as the number of cases identified with disease-associated genes based on the clinician's assessment. Negative cases are defined as the opposite. Positive cases (as judged by data analysts) are defined by the number of cases identified with suspected disease-related genes by analysts by using the whole exome sequencing data.

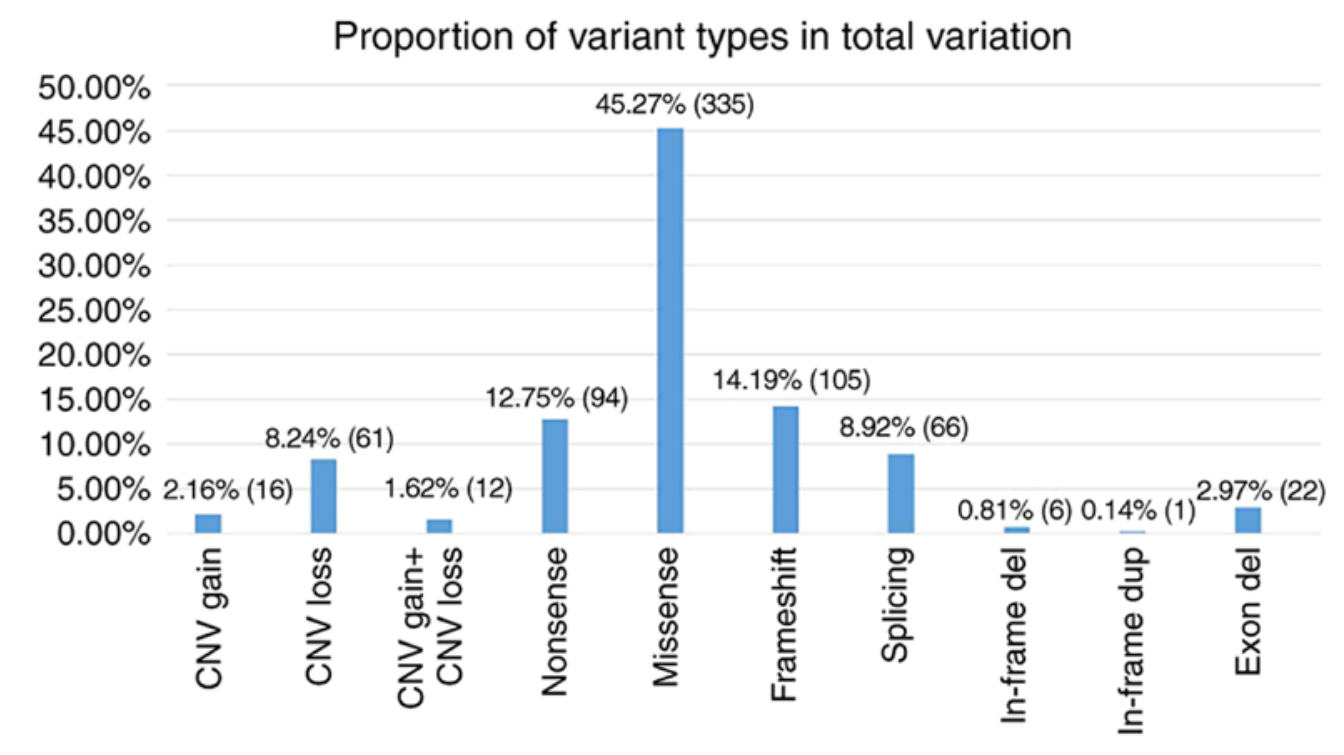

Figure 3. Proportion of variant types in the total variations. CNV, copy number variant; del, deletion; dup, duplication.

approved $\sim 400$ types of orphan drugs, but these drugs have limited efficacy and are suitable for treatment of only $\sim 5.00 \%$ of rare diseases. Most rare diseases may only rely on symptomatic or placebo treatments, as the causes of most of these genetic diseases still remain elusive (22-24). In recent years, with the advancement of precision medicine and the rapid development of large-scale parallel sequencing technology, the application of WES has increased, not only for research purposes but also for clinical diagnosis. The effectiveness of WES has been well documented for certain diseases, such as those relating to the nervous system, dermatology and seizures (25-27). This may help to elucidate the pathogenesis of these diseases.

In general, genetic variations may affect biological function by enhancing (e.g., dose effects and alterations of transcriptional activity) or decreasing (e.g., haplo-insufficiency and dominant-negative mutation) the function of key genes (28).

WES was performed on all 1,360 patients of the present study. The examination and analytical processes are depicted in a schematic in Fig. 4, including those performed prior to, during and after WES. The overall positive rate obtained with 


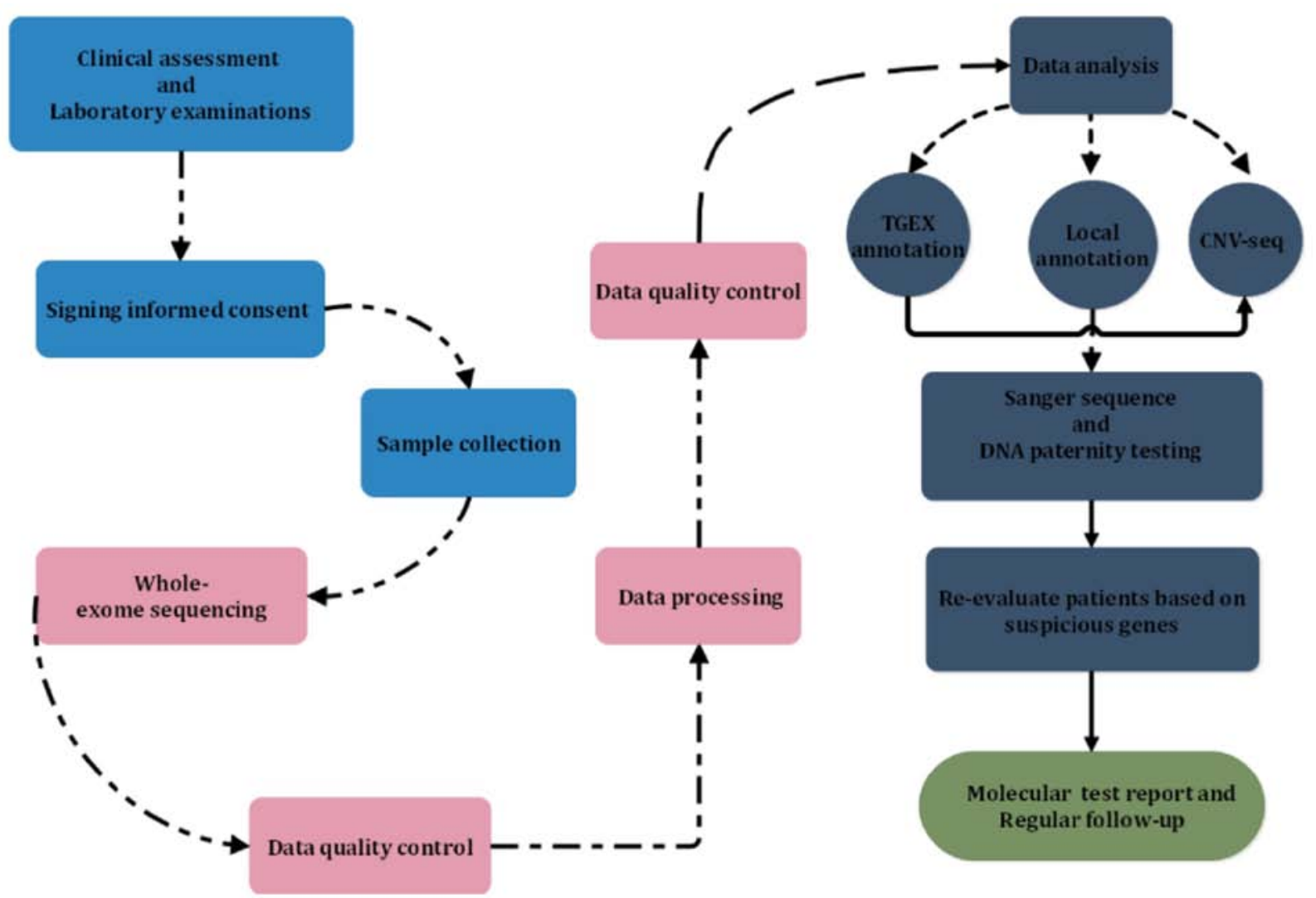

Figure 4. Whole-exome sequencing analysis flow. CNV, copy number variant.

this protocol was $47.05 \%$, which was slightly higher than that for a previous case series, in which yields of 25.00-26.00\% were reported (29) and it was higher than the positive rate of other genetic testing methods, such as whole-genome sequencing analysis (34.00-42.00\%), karyotype analysis (4.20-10.00\%), chromosome microarray analysis (6.50-20.00\%) and fluorescence in situ hybridization analysis (3.00\%) (30-36). Among them, the trio-samples positive rate was $6.29 \%$ higher than the rate obtained for single samples, and this was similar to the results obtained from a previous study (37).

Analysis of trio-samples may not only identify the mutations associated with the disease detected based on the clinical manifestations but may also, in turn, be compared and evaluated from the severity of the mutations to the clinical performance of the patient. At the same time, it is possible to rapidly eliminate irrelevant variations by using the genetic model, allowing for the identification of suspected disease-causing genes, ultimately increasing the positive detection rate and efficiency. In addition, trios facilitate the detection of de novo variants and allow for appraisal of compound heterozygous variants during interpretation (rather than during the confirmatory testing process). When WES was performed, factors to be considered by clinicians included cost, time-to-result and the presence of consanguinity or family history. For certain diseases that are rapidly progressing and have serious clinical symptoms, this study would suggest that priority should be given to performing WES where trios are available.

In the present study, the major clinical manifestations of 1,360 patients were classified into 20 main categories in order to determine which symptoms contributed the most to the positive rate of WES. Among the 20 classifications, the positive rate of audio or visual abnormalities was the highest (58.51\%), followed by abnormality of the head or neck $(55.52 \%)$ and global developmental delay (54.84\%). The manifestations with the lowest positive rate were abnormalities of the digestive system (26.92\%). A total of 52 patients with abnormality of the digestive system were analyzed, revealing that the major symptoms were diarrhea, constipation, vomiting and gastro-esophageal reflux. The age of the affected children ranged from several days to several months. At this stage, the appearance of such symptoms was mainly related to the developmental characteristics of the infants with an immature digestive system and minimal establishment of microflora. Therefore, it would be difficult to adapt to changes in the quality and quantity of food. In addition, those subjects are likely to have low body immunity and incorrect feeding regimens employed by certain parents as well as other factors may lead to these symptoms. However, such symptoms should not be ignored during the analysis, as they may also be characteristic of certain hereditary diseases, such as Hirschsprung disease 2 [online mendelian inheritance in man (OMIM) ID, 600155].

The three most common variations were missense, frameshift and nonsense mutations and the least common ones were in-frame duplications. It is worth noting that of the 604 positive cases, 22 had exon deletions, such as ASCC1 (activating signal cointegrator 1 complex subunit 1) c.932C $>\mathrm{G}$ (p.Ser311Ter) or exon5 deletion. These small deletions should always be noted, as they may be missed during routine and low-depth $\mathrm{CNV}$ analyses. During the analysis of an autosomal recessive disease, when one mutation is found in a certain gene, which is relevant 
to the clinical symptoms, we recommended that the exons of this particular gene should be carefully viewed using IGV software.

WES may help clinicians to determine the cause of the disease and can also influence the treatment strategy, such as pyridoxine-dependent epilepsy caused by the ALDH7A1 gene (aldehyde dehydrogenase 7 family member A1; OMIM ID, 266100). Patients with this type of epilepsy are generally insensitive to treatment with anticonvulsants but may be treated effectively with large doses of pyridoxine (vitamin B6). WES, which Raffan and Semple (38) called 'game-changing technology' in 2011, is becoming an essential tool for clinicians dealing with rare and common genetic disorders.

It may be assumed that due to the maturity of WES technology and the standardization of the process, the data analysis performed by different analysts according to standardized procedures does not exhibit any obvious differences in the results obtained. In order to avoid the influence of subjective factors, indicators of different diseases were quantified in the present study, so as to minimize variations caused by those subjective factors. For instance, short stature was evaluated according to items including the standard deviation, the annual increase in height and growth hormone stimulation test results.

There are numerous challenges associated with WES. First of all, there is an increasing number of non-paternity infants (from donor gametes), which makes it difficult to identify and interpret suspected pathogenic variants. Furthermore, there are complex issues surrounding genetic counseling associated with WES (e.g., variants of unknown significance). Finally, establishing a close cooperation network between analysts and clinicians in the clinical environment may be challenging.

In summary, there are numerous difficulties and challenges that require to be overcome to successfully apply WES sequencing technology in the fields of healthcare and disease prevention. Fortunately, the situation is constantly improving. In the foreseeable future, it is anticipated that WES will become a valuable and possibly even a first-line diagnostic tool in the clinical setting for the diagnosis of complex genetic diseases.

\section{Acknowledgements}

The authors would like to thank Dr Dev Sooranna (Division of Cancer, Academic Department of Obstetrics \& Gynaecology, Imperial College, London, SW10 9NH, UK) for editing the manuscript.

\section{Funding}

This study was supported by the Guangxi Zhuang Region Health Department (grant no. Z20190311).

\section{Availability of data and materials}

The datasets used and/or analyzed during the current study are available from the corresponding author on reasonable request.

\section{Authors' contributions}

QZ and ZQ designed the study and analyzed the relevant literature. ZQ wrote the manuscript and prepared the figures. SY and $\mathrm{XZ}$ processed the data, HW and JS collected the blood samples and performed the DNA extraction experiments. QZ, $\mathrm{ZQ}, \mathrm{XZ}$ and JS were involved in performing the WES and the patient examinations. QZ and SY confirm the authenticity of all the raw data. All the authors read and approved the final manuscript.

\section{Ethics approval and consent to participate}

The study was approved by the Ethics Review Committee of the Maternal and Child Health Hospital of Guangxi (Nanning, Guangxi) and was performed in accordance with the approved guidelines.

\section{Patient consent for publication}

Not applicable.

\section{Competing interests}

The authors declare that they have no competing interests.

\section{References}

1. Yu M, Ping Z, Zhang S, He Y, Dong R and Guo X: The survey of birth defects rate based on birth registration system. Chin Med J (Engl) 128: 7-14, 2015.

2. Cai L, Zheng LA and He L: The forty years of medical genetics in China. J Genet Genomics 45: 569-582, 2018.

3. Zhang Q, He L and Shen YP: The arrival of the clinical whole genome era. Zhonghua Er Ke Za Zhi 57: 401-404, 2019 (In Chinese).

4. Cram DS and Zhou D: Next generation sequencing: Coping with rare genetic diseases in China. Intractable Rare Dis Res 5: 140-144, 2016.

5. Zhao X, Wang P, Tao X and Zhong N: Genetic services and testing in China. J Community Genet 4: 379-390, 2013.

6. Ng SB, Turner EH, Robertson PD, Flygare SD, Bigham AW, Lee C, Shaffer T, Wong M, Bhattacharjee A, Eichler EE, et al: Targeted capture and massively parallel sequencing of 12 human exomes. Nature 461: 272-276, 2009.

7. Dillon OJ, Lunke S, Stark Z, Yeung A, Thorne N, Melbourne Genomics Health Alliance; Gaff C, White SM and Tan TY: Exome sequencing has higher diagnostic yield compared to simulated disease-specific panels in children with suspected monogenic disorders. Eur J Hum Genet 26: 644-651, 2018.

8. Chen P, He G, Zou X, Zhang X, Li J, Wang Z, Gao H, Luo L, Zhang Z, Yu J and Han Y: Genetic diversities and phylogenetic analyses of three Chinese main ethnic groups in southwest China: A Y-Chromosomal STR study. Sci Rep 8: 15339, 2018.

9. Business Wire: The Maternal and Children Health Hospital of Guangxi Zhuang Autonomous Region and LifeMap Sciences Join Hands to Improve Diagnosis for Rare Diseases. Business Wire, Inc., 2018. https://www.businesswire.com/ news/home/20180417005741/en/Maternal-Children-HealthHospital-Guangxi-Zhuang-Autonomous.\%20Journal. Accessed April 17, 2018.

10. Hu P, Qin YH, Jing CX, Lu L, Hu B and Du PF: Does the geographical gradient of ApoE4 allele exist in China? A systemic comparison among multiple Chinese populations. Mol Biol Rep 38: 489-494, 2011.

11. Li H and Durbin R: Fast and accurate long-read alignment with Burrows-Wheeler transform. Bioinformatics 26: 589-595, 2010.

12. Van der Auwera GA, Carneiro MO, Hartl C, Poplin R, Angel GD, Levy-Moonshine A, Jordan T, Shakir K, Roazen D, Thibault J, et al: From FastQ data to high confidence variant calls: The genome analysis toolkit best practices pipeline. Curr Protoc Bioinformatics 43: 11.10.11-11.10.33, 2013.

13. Abel HJ, Duncavage EJ, Becker N, Armstrong JR, Magrini VJ and Pfeifer JD: SLOPE: A quick and accurate method for locating non-SNP structural variation from targeted next-generation sequence data. Bioinformatics 26: 2684-2688, 2010. 
14. Yoon S, Xuan Z, Makarov V, Ye K and Sebat J: Sensitive and accurate detection of copy number variants using read depth of coverage. Genome Res 19: 1586-1592, 2009.

15. Li C and Hung Wong W: Model-based analysis of oligonucleotide arrays: Model validation, design issues and standard error application. Genome Biol 2: RESEARCH0032, 2001.

16. Li X, Chen S, Chen F, Xie W, Wang J, Wang J, Yang H and Zhang X: A kind of copy number mutation detection method and system. CN Patent CN104221022B. Filed April 5, 2014; issued November 21, 2017.

17. Li Q and Wang K: InterVar: Clinical interpretation of genetic Variants by the 2015 ACMG-AMP guidelines. Am J Hum Genet 100:267-280, 2017

18. Editorial Board, Chinese Journal of Pediatrics: Consensus recommendations for the clinical application of genetic testing for children's genetic diseases. Zhonghua Er Ke Za Zhi 57: 172-176, 2019 (In Chinese)

19. Richards S, Aziz N, Bale S, Bick D, Das S, Gastier-Foster J, Grody WW, Hegde M, Lyon E, Spector E, et al: Standards and guidelines for the interpretation of sequence variants: A joint consensus recommendation of the American college of medical genetics and genomics and the association for molecular pathology. Genet Med 17: 405-424, 2015.

20. Jamuar SS and Tan EC: Clinical application of next-generation sequencing for Mendelian diseases. Hum Genomics 9: 10, 2015.

21. Harris J: Germline manipulation and our future worlds. Am J Bioeth 15: 30-34, 2015.

22. Baird PA, Anderson TW, Newcombe HB and Lowry RB: Genetic disorders in children and young adults: A population study. Am J Hum Genet 42: 677-693. 1988.

23. Valdez R, Grosse SD and Khoury MJ: The need for a next-generation public health response to rare diseases. Genet Med 19: 489-490, 2016.

24. Zhao M and Wei DQ: Rare Diseases: Drug discovery and informatics resource. Interdiscip Sci 10: 195-204, 2018.

25. Reches A, Hiersch L, Simchoni S, Barel D, Greenberg R, Ben Sira L, Malinger G and Yaron Y: Whole-exome sequencing in fetuses with central nervous system abnormalities. J Perinatol 38 : 1301-1308, 2018

26. Caylor RC, Grote L, Thiffault I, Farrow EG, Willig L, Soden S, Amudhavalli SM, Nopper AJ, Horii KA, Fleming E, et al: Incidental diagnosis of tuberous sclerosis complex by exome sequencing in three families with subclinical findings. Neurogenetics 19: 205-213, 2018.

27. Kobayashi Y, Tohyama J, Kato M, Akasaka N, Magara S, Kawashima H, Ohashi T, Shiraishi H, Nakashima M, Saitsu H and Matsumoto N: High prevalence of genetic alterations in early-onset epileptic encephalopathies associated with infantile movement disorders. Brain Dev 38: 285-292, 2016.

28. Jackson M, Marks L, May GHW and Wilson JB: The genetic basis of disease. Essays Biochem 62: 643-723, 2018
29. Retterer K, Juusola J, Cho MT, Vitazka P, Millan F, Gibellini F, Vertino-Bell A, Smaoui N, Neidich J, Monaghan KG, et al: Clinical application of whole-exome sequencing across clinical indications. Genet Med 18: 696-704, 2016.

30. Stavropoulos DJ, Merico D, Jobling R, Bowdin S, Monfared N, Thiruvahindrapuram B, Nalpathamkalam T, Pellecchia G, Yuen RKC, Szego MJ, et al: Whole genome sequencing expands diagnostic utility and improves clinical management in pediatric medicine. NPJ Genom Med 1: 15012, 2016.

31. Gudbjartsson DF, Helgason H, Gudjonsson SA, Zink F, Oddson A, Gylfason A, Besenbacher S, Magnusson G, Halldorsson BV, Hjartarson E, et al: Large-scale whole-genome sequencing of the Icelandic population. Nat Genet 47: 435-444, 2015.

32. Dai R, Yu Y, Xi Q, Hu X, Zhu H, Liu R and Wang R: Prenatal diagnosis of 4953 pregnant women with indications for genetic amniocentesis in Northeast China. Mol Cytogenet 12: 45, 2019.

33. Shaffer LG, Bejjani BA, Torchia B, Kirkpatrick S, Coppinger J and Ballif BC: The identification of microdeletion syndromes and other chromosome abnormalities: Cytogenetic methods of the past, new technologies for the future. Am J Med Genet C Semin Med Genet 145C: 335-345, 2007.

34. Shaffer LG, Rosenfeld JA, Dabell MP, Coppinger J, Bandholz AM, Ellison JW, Ravnan JB, Torchia BS, Ballif BC and Fisher AJ: Detection rates of clinically significant genomic alterations by microarray analysis for specific anomalies detected by ultrasound. Prenat Diagn 32: 986-995, 2012.

35. Miller DT, Adam MP, Aradhya S, Biesecker LG, Brothman AR, Carter NP, Church DM, Crolla JA, Eichler EE, Epstein CJ, et al: Consensus statement: Chromosomal microarray is a first-tier clinical diagnostic test for individuals with developmental disabilities or congenital anomalies. Am J Hum Genet 86: 749-764, 2010.

36. Ravnan JB, Tepperberg JH, Papenhausen P, Lamb AN, Hedrick J, Eash D, Ledbetter DH and Martin CL: Subtelomere FISH analysis of 11688 cases: An evaluation of the frequency and pattern of subtelomere rearrangements in individuals with developmental disabilities. J Med Genet 43: 478-489, 2006.

37. Yates CL, Monaghan KG, Copenheaver D, Retterer K, Scuffins J, Kucera CR, Friedman B, Richard G and Juusola J: Whole-exome sequencing on deceased fetuses with ultrasound anomalies: Expanding our knowledge of genetic disease during fetal development. Genet Med 19: 1171-1178, 2017.

38. Raffan E and Semple RK: Next generation sequencing-implications for clinical practice. Br Med Bull 99: 53-71, 2011.

This work is licensed under a Creative Commons Attribution-NonCommercial-NoDerivatives 4.0 International (CC BY-NC-ND 4.0) License. 